\title{
Digital image classification by optimised fuzzy system
}

\author{
Mehdi Ramezanifard ${ }^{1}$, B. S. Mousavi ${ }^{2}$ \\ ${ }^{1}$ Electrical Engineering Department, Velayat University, Iran \\ ${ }^{2}$ Department of Electrical Engineering, Hatef Higher Education Institute, Iran
}

\begin{tabular}{l}
\hline \hline Article Info \\
\hline Article history: \\
Received Nov 7, 2018 \\
Revised Feb 15, 2019 \\
Accepted Feb 27, 2019 \\
\hline
\end{tabular}

Keywords:

Fuzzy inference system

Genetic algorithm

Image classification

Zernike moments

\begin{abstract}
Since image classification is a complex process that may be affected by many factors, it is a challenging problem of computer vision. This study reports a fuzzy system to semantic image classification. As it is a complex task, various information of digital image, including: three color space components and two Zernike moments with different order are gathered and utilized as an input of fuzzy inference system to materialize a robust rotation/lighting condition and size invariant image classifier. For better performance, all the membership functions are optimized by genetic algorithm after empirically design stage. $93.07 \%$ and $95.25 \%$ classification rates empirically design and optimized systems confirm the reliability of proposed method in different image conditions given in this contribution.
\end{abstract}

Copyright $@ 2019$ Institute of Advanced Engineering and Science. All rights reserved.

\section{Corresponding Author:}

Mehdi Ramezanifard,

Electrical Engineering Department,

Velayat University,

Iranshahr, Iran.

Email: m.ramezanifard@ velayat.ac.ir

\section{INTRODUCTION}

Semantic labeling of digital images by classification is applied to content-based image retrieval and image annotation [1]. However, it is a difficult task to group images into semantic classes because of different colors and textures in various image classes [2]. In fact, today Vision and Pattern Recognition problems such as face recognition, fingerprinting identification, image categorization, and DNA sequencing often have an arbitrarily large number of properties to consider. To solve such complex problems using just one feature descriptor is a difficult task and feature fusion may become mandatory. Normal feature fusion is nearly effective for many problems. However, it can result in unexpected classification results when the different features are not properly combined. In addition, it has the drawback of increasing the dimensionality which might require more training data.

To cope with these problems, this paper introduces an optimized fuzzy system, based on color and texture information. This approach is more flexible because of fuzzy concepts and it is more adequate to some problems than a naive method. Our Mamdani fuzzy inference system use three components of color space and two Zernike moment as inputs, where all these features are simply concatenated and fed to fuzzy classification algorithm and, three outputs shows the probability of belonging to three different image classes. Besides that, the presented technique is amenable to deal with much more digital image classes.

Besides, introducing new approach, proposed method is applied in two different color spaces and obtained results are discussed. For more information, one may consult. Generally, the objectives of this study are: (i) to evaluate fuzzy inference system and Zernike moments for image classification aim, (ii) to introduce new method of fuzzy system optimization based on genetic algorithm and (iii) to compare two color spaces.

In Section 2, Zernike Moments are discussed. Section 3 elaborates the proposed classification method. Results and conclusion are presented in Sections 4 and 5. 


\section{ZERNIKE MOMENTS}

$\mathrm{Hu}[3]$ showed the classical moment's application to two dimensional images in the early sixties. He tested their validity using a simple experiment to recognize written characters. Hu was only concerned with images without noise, but further work showed that traditional moment performance degrades where the view is occluded or noisy [4]. A survey of moment based techniques with respect to computer application details many of the current techniques regarding representation and recognition [5].

Zernike moments are a class of orthogonal moments and have been shown effective in terms of image representation. Zernike moments can be easily constructed to an arbitrary order. Although higher order moments carry more fine details of an image, they are also more susceptible to noise. Therefore, we have experimented with different orders of Zernike moments to determine the optimal order for our problem. The Zernike polynomials are a set of complex, orthogonal polynomials defined over the interior of a unit circle $\mathrm{x} 2+\mathrm{y} 2=1[6]$.

$$
\begin{aligned}
& V_{n m}(x, y)=V_{n m}(\rho, \theta)=R_{n m}(\rho) e^{j m \theta} \\
& R_{n m}(\rho)=\sum_{s=0}^{\frac{n-|m|}{2}}(-1)^{s} \frac{(n-s) !}{s !\left[\frac{n+|m|}{2}-s\right] !\left[\frac{n-|m|}{2}-s\right] !} \rho^{n-2 s}
\end{aligned}
$$

where, $\mathrm{n}$ is a non-negative integer, $\mathrm{m}$ is an integer such that $\mathrm{n}-|\mathrm{m}|$ is even and $|\mathrm{m}| \leq \mathrm{n}, \rho=\sqrt{x^{2}+y^{2}}$, and $\theta=\tan -1(\mathrm{y} / \mathrm{x})$.

Projecting the image function onto the basis set, the Zernike moment of order $\mathrm{n}$ with repetition $\mathrm{m}$ is:

$$
A_{n m}=\frac{n+1}{\pi} \sum_{x} \sum_{y} f(x, y) V_{n m}, x^{2}+y^{2} \leq 1
$$

As the Zernike moments are calculated using the radial polynomials, they have inherent rotation invariant property. It has been shown in [7] that the Zernike moments on a rotated image differ from those of the original un-rotated image in phase shifts, but not in magnitudes. Therefore $|\mathrm{Anm}|$ can be used as a rotation invariant feature of the image function. Since $A n,-m=A n m$, and therefore $|A n,-m|=|A n m|$, we will use only $|\mathrm{Anm}|$ for features. Since $|\mathrm{A} 00|$ and $|\mathrm{A} 11|$ are the same for all of the normalized symbols, they will not be used in the feature set. Therefore the extracted features of the order $n$ start from the second order moments up to the nth order moments.

\section{PROPOSED ALGORITHM}

Moment descriptors have been studied for image recognition and computer vision since the 1960s. Teague first introduced the use of Zernike moments to overcome the shortcomings of information redundancy present in the popular geometric moments. Here, we use two order of Zernike moments beside color information to materialize a robust semantic color image classification. Better evaluation and comparing, two empirical and optimized systems are designed for HSV, color space. Details of learning and designing process are presented in following sections.

\subsection{Empirically Design Classification System}

As group images into semantic classes is complex and should be applicable for digital images in various lighting / resolution / size and environmental conditions, using fuzzy inference system is a proper offer to overcome this complexity and improve system flexibility. In designing FIS three major steps should be followed:

a) The number of inputs and designing their MFs

b) The number of outputs and designing their MFs

c) Defining rules to connect inputs and outputs

Five inputs are considered for FIS. Three of them are three color spaces components. These inputs provides color and intensity information of digital color image. To increase system accuracy a Zernike moments descriptor information is applied as a two other inputs. These inputs add image texture properties to our system. Two dimension images are needed to calculate Zernike moments. We use intensity value image 
of color image to this aim. Figure 1 shows an RGB, HSV and obtained intensity images. The center of image is considered as a center of unit circle and image is mapping inside it. Using (3) Zernike moments could be calculated. To have both low and high moments information, we use 4th and 40th magnitude (at $\theta=30^{\circ}$ ) moments as fourth and fifth inputs, respectively.

The number of outputs should be equal to image classes. In this work we form a database contains three different classes. They include: Face, See and Mountains images. So, we have three outputs where each of them reveals the probability of belonging to special class. Some examples of our database images are shown in Figure 2.

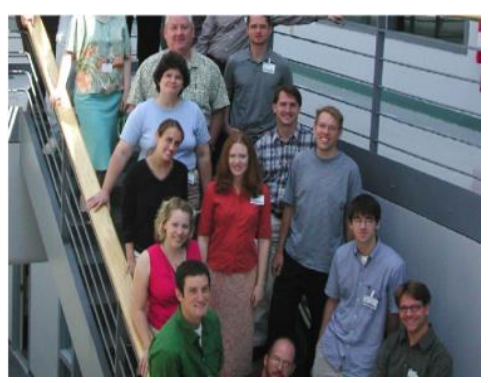

(a)

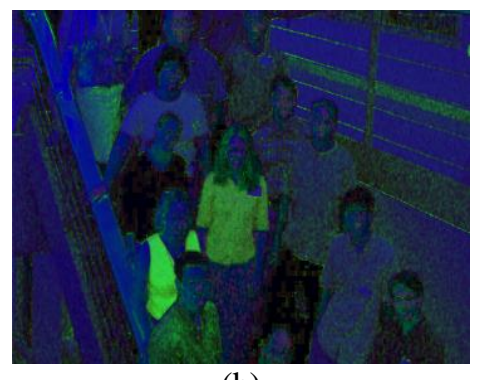

(b)

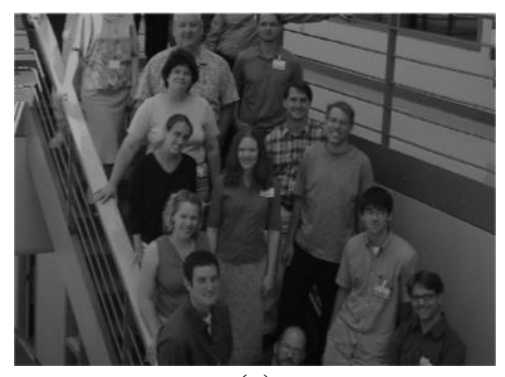

(c)

Figure 1. (a) RGB image, (b) HSV image, (c) Intensity image
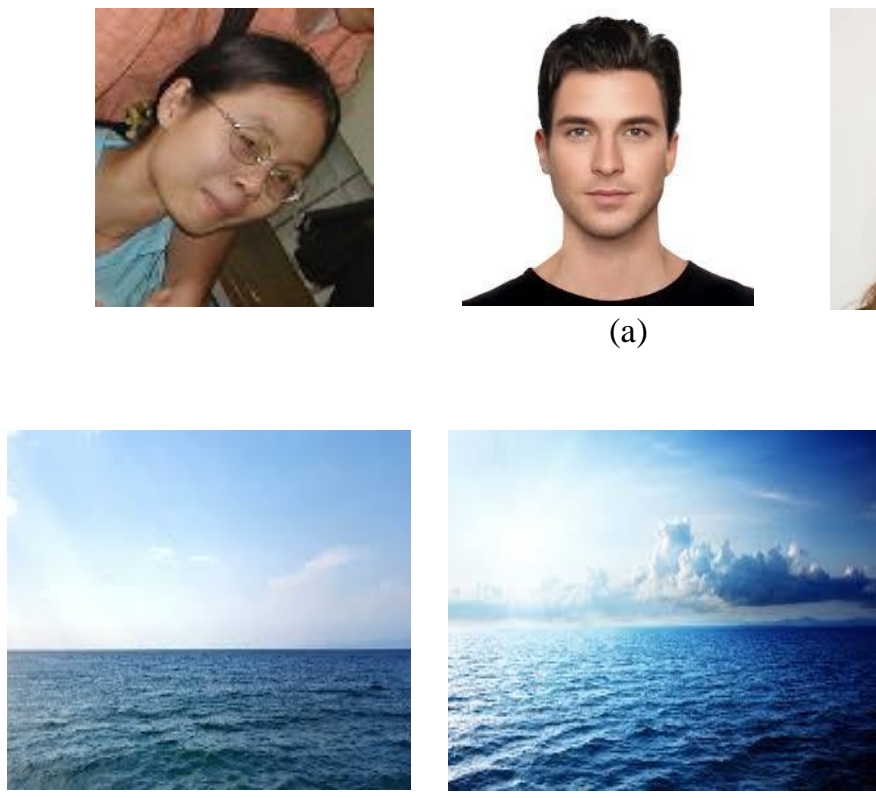

(b)

(a)

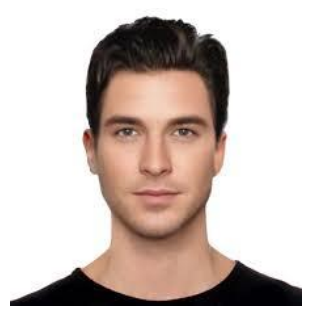

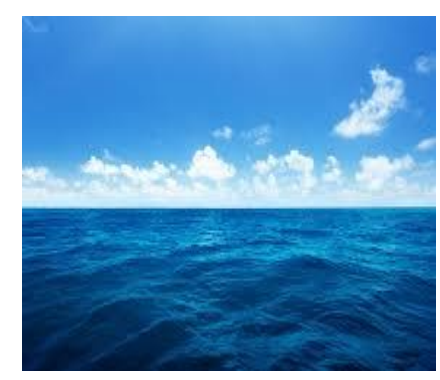
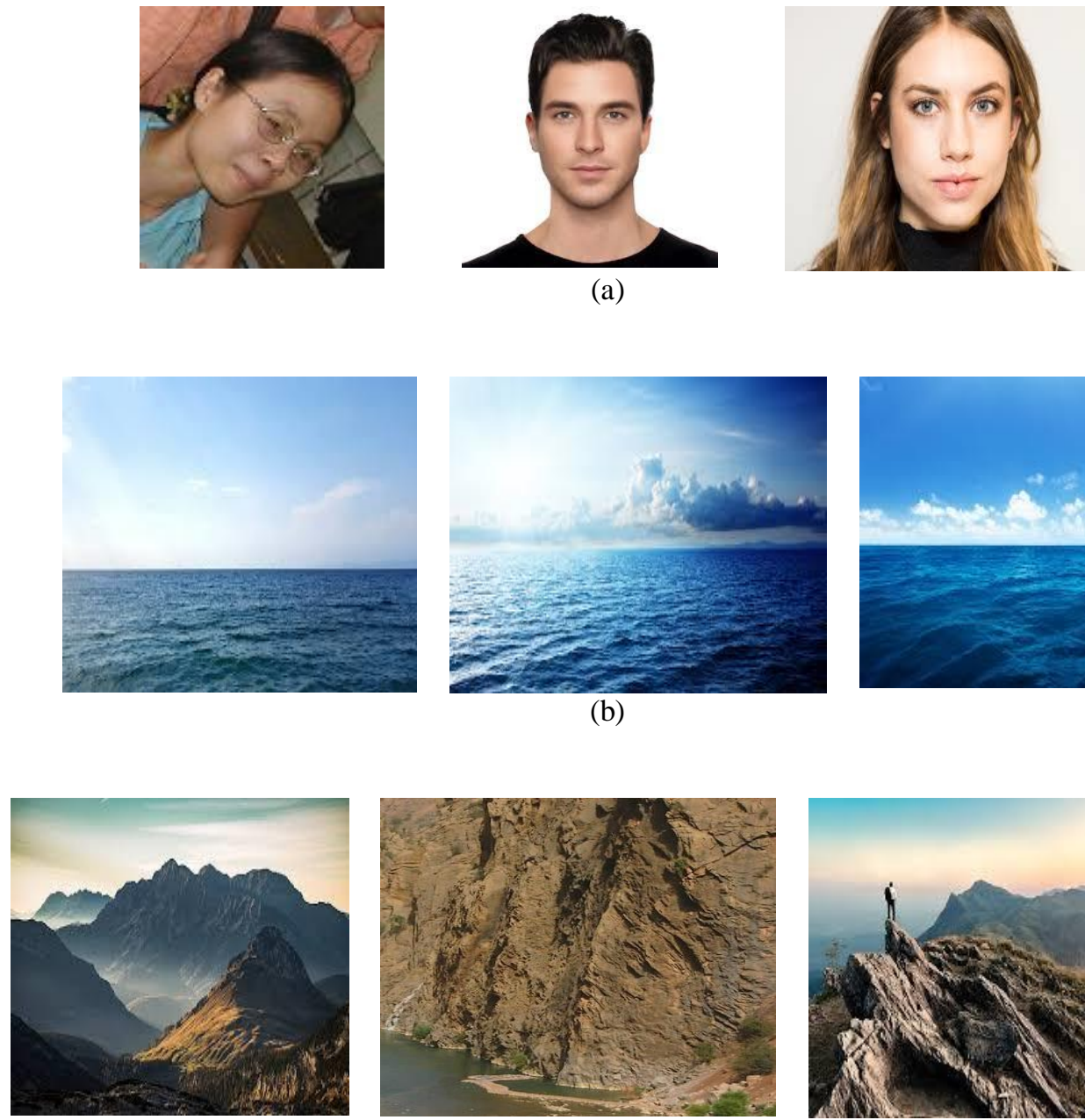

(c)

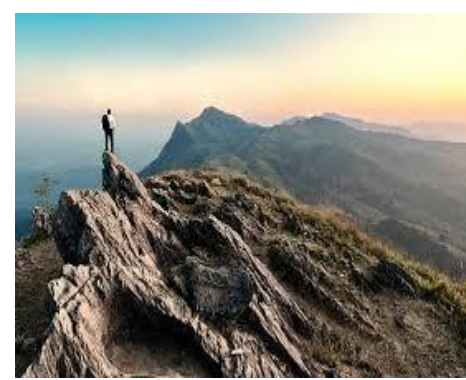

nax

Figure 2. Sample images of collected database, (a) face, (b) Sea and (c) Mountain classes 
Mamdani fuzzy inference system is applied to make decision. Both inputs and outputs should be interpreted by appropriate MFs. In this stage, after various investigations, they have achieved using experimental knowledge. 30 rules are defined and centroid method [8] is utilized to have crisp outputs.

This system uses a sample color image as input and there are three outputs show the probability of belonging to three different image classes. The image is labelled as a class which reach higher probability. If all outputs be less than $50 \%$, the input image would belong to none of considered image classes. Figure 3 show inputs and outputs MFs.

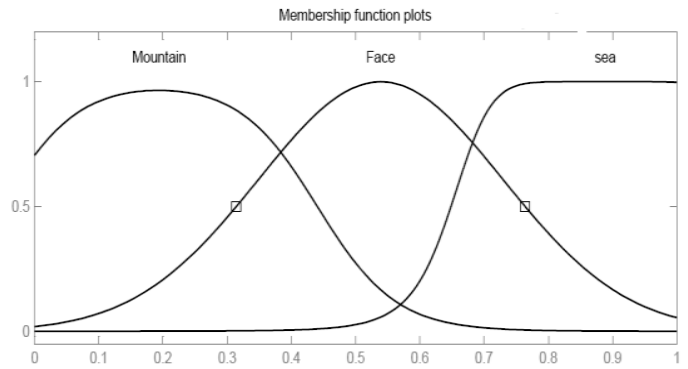

(a)

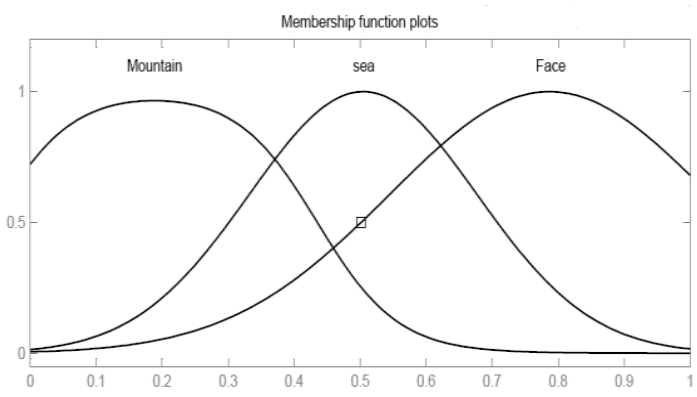

(c)

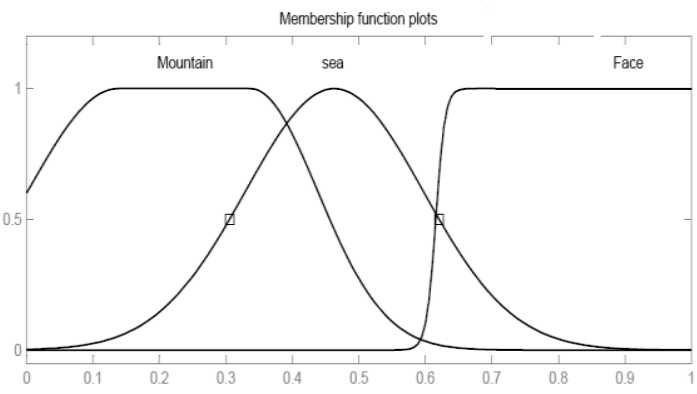

(e)

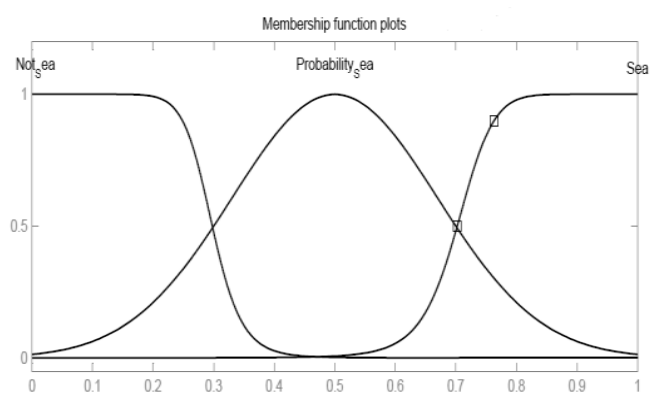

(g)

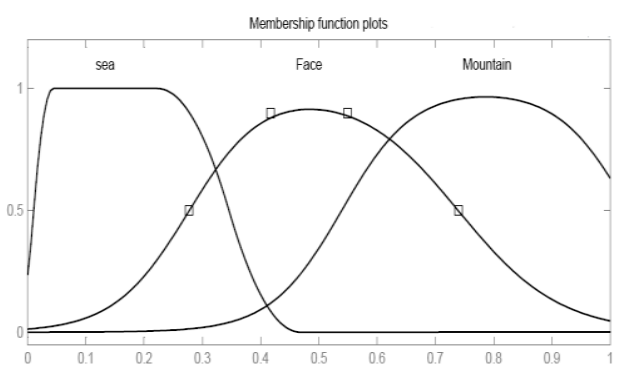

(b)

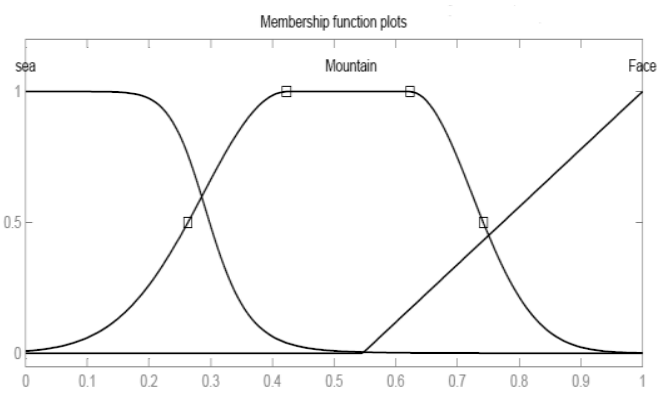

(d)

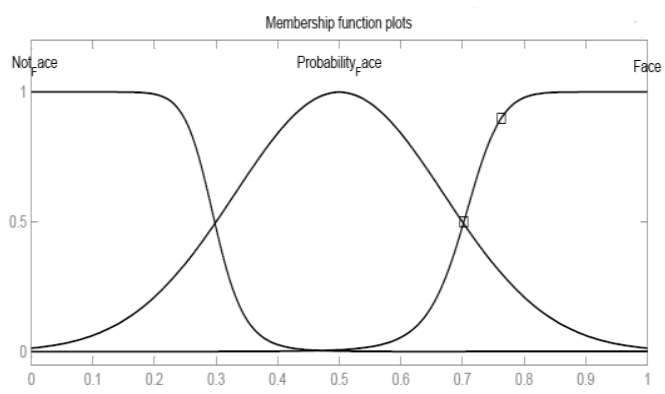

(f)

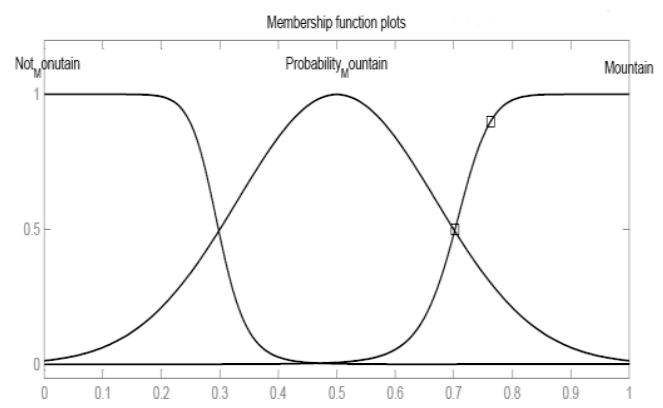

(h)

Figure 3. (a)-(e) inputs and (f)-(h) outputs MFs in HSV color space 


\subsection{Optimization by GA}

Fuzzy inference system is reliable method. Steel, it is often difficult for a human expert to define the fuzzy sets and fuzzy rules. GAs has proven to be a useful method to optimize membership functions of fuzzy sets [9]. GA parameters are selected as follows:

Initial population $=100$

Crossover coefficients $=0.7$

Mutation coefficient (mutation is done in an un-uniform) $=0.05$

Migration coefficient $=0.15$

Our empirical knowledge is utilized to reduce the searching space and expedite the process. The MFs' shapes are chosen according to previous designed system and the parameters of MFs are utilized as the inputs of the GA, whose fitness function attempts to maximize the output probability for the class that input image belongs to it. Figure 4 illustrate optimized MFs.

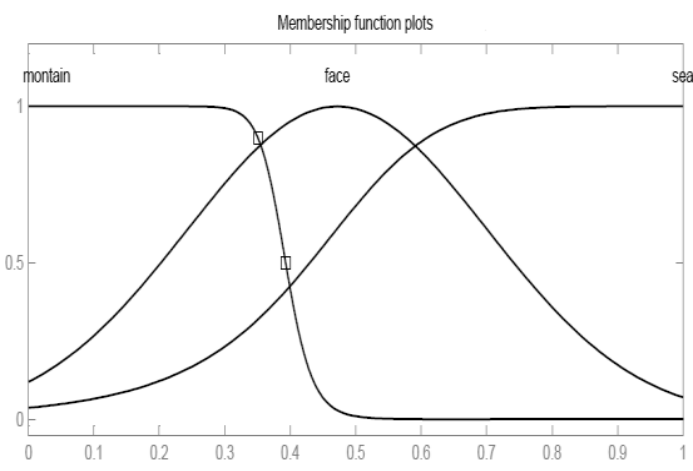

(a)

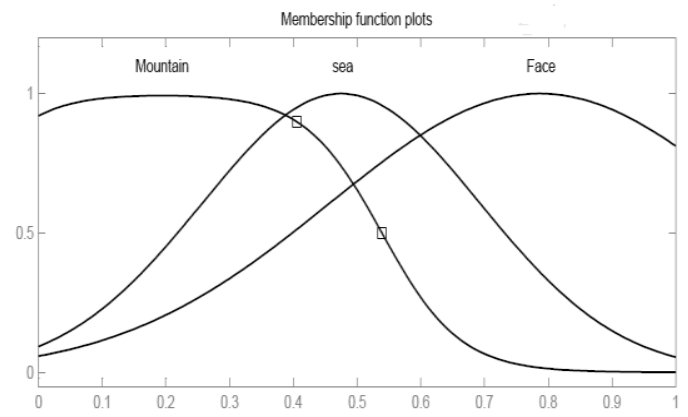

(c)



(e)

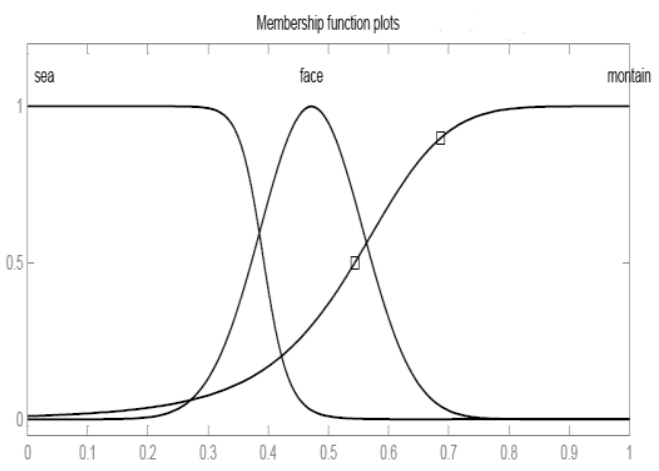

(b)

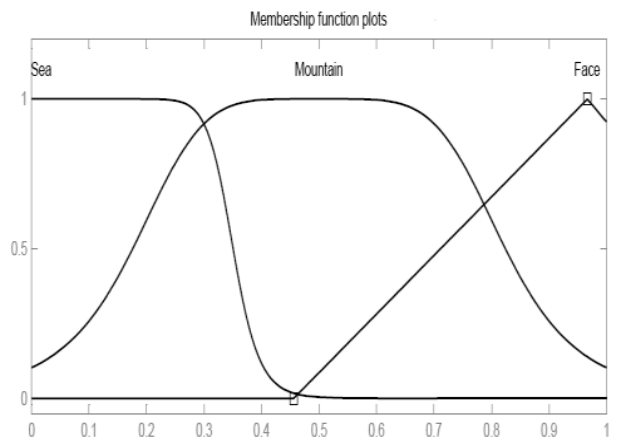

(d)

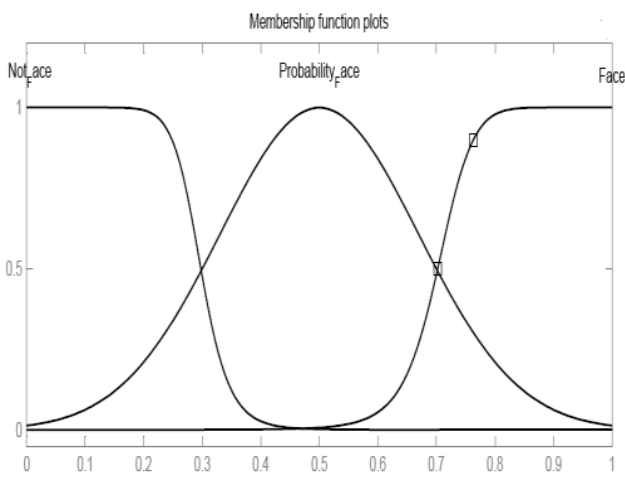

(f) 


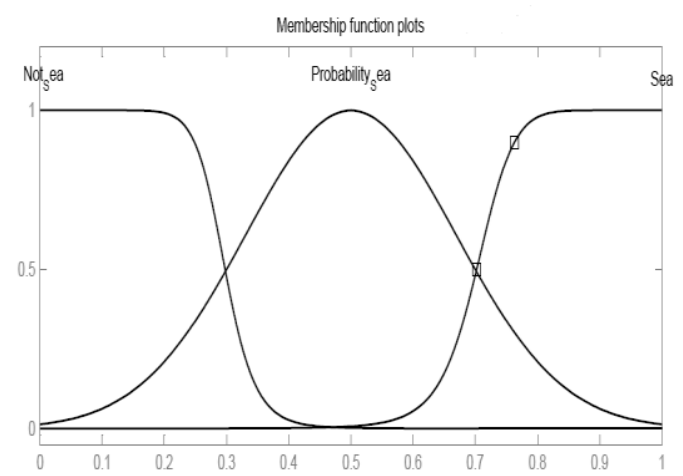

(g)

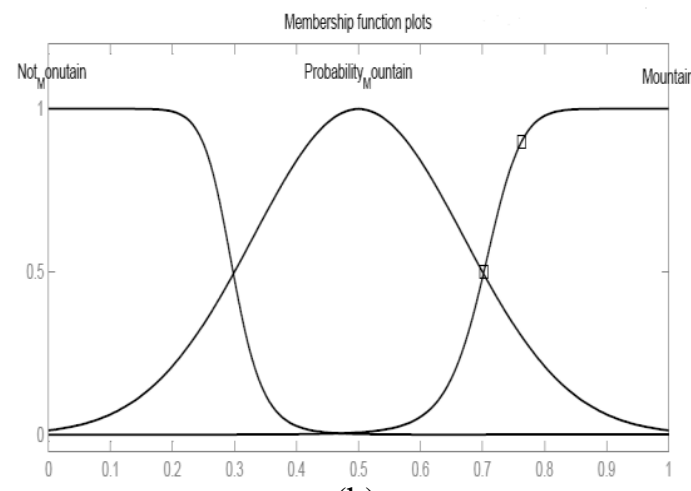

(h)

Figure 4. (a)-(e) inputs and (f)-(h) outputs optimised MFs in HSV color space

\section{RESULTS}

To evaluate designed systems performance, we gather a proper image database. This database contains 40 face images, 50 sea images, 50 mountains images and 50 various images that they do not lie in three previous groups. Our images are from 120x132 to 560x350 in size. Different lightening condition and rotated images are considered in this dataset. Both empirically design and optimised systems, called System 1 and System 2 respectively, are applied and achieved results are presented in Table 1.

Table 1. Obtained Classification Rates $(\%)$

\begin{tabular}{ccccccccc}
\hline & \multicolumn{3}{c}{ System 1 } & \multicolumn{5}{c}{ System 2 } \\
& Face & Sea & Mountain & Total & Face & Sea & Mountain & Total \\
\hline Rate & 90 & 95 & 90 & 93.05 & 92.5 & 97.5 & 95 & 95.25 \\
\hline
\end{tabular}

With investigating obtained results three image classes two designed system could be compared. In all cases sea class shows the best result. In the images of this group both color and texture information are approximately constant. So, based on these properties, it is expectable that we have the most classification rate. Although mountains images have similar color, their texture differs from each other. As face images contain wide range of skin color and various textures, because of different face expression, decrease in classification rate is unavoidable. Considering face images condition, obtained results are still acceptable.

\section{CONCLUSION}

This paper has offered a new semantic image classifier. Beside color information the image texture properties were applied by computing Zernike moments. All information was used as inputs of fuzzy inference system for making decision and classification. In addition, after empirically system design, all membership functions are optimized by genetic algorithm and best shape of them were achieved. Obtained results proved much better performance for optimized system. Suggested method to optimize fuzzy system is a novel way, could be applied to design membership functions in any arbitrary fuzzy system, generally [10]-[12].

\section{REFERENCES}

[1] V.M. Figueiredo, A.K. Jain, H.-J. Zhang, "Image classification for content-based indexing," IEEE Transactions on Image Process, vol. 10, pp. 117-130, 2001.

[2] D. Wang, J. Shik Lim, M.-M. Han, Byung-Wook Lee, "Learning similarity for semantic images classification," Neurocomputing, vol.67, pp. 363-368, 2005.

[3] M. Hu. "Visual Pattern Recognition by Moment Invariants," IRE Trans. on Information Theory, IT-8, pp. $179-187,1962$.

[4] C. Teh and R. T.Chin., "On image analysis by the method of moments," IEEE Trans. PAMI, vol. 10, pp. 496-513, 1988.

[5] R. J. Prokop and A. P. Reeves., "A survey of moment-based techniques for un-occluded object representation and recognition," CVGIP GMIP, vol. 54, pp. 438-460, 1992. 
[6] Hyoung-Joon Kim and Whoi-Yul Kim, "Eye Detection in Facial Images Using Zernike Moments with SVM," ETRI Journal, Vol. 30, 2008.

[7] Khotanzad, A. and Hong, Y.H., "Rotation Invariant Image Recognition using Features Selected via a Systematic Method," Pattern Recognition, vol. 23, pp. 1089-1101, 1990.

[8] S.N. Sivanandum, S. Sumathi, S.N. Deepa, "Introduction to Fuzzy logic using MATLAB," Springer-Verlag Berlin Heidelberg, 2007.

[9] S.N. Sivanandam S.N. Deepa, "Introduction to Genetic Algorithms", Springer-Verlag Berlin Heidelberg, pp. 165-209, 2008.

[10] Hussain Attia, Fernando Gonzalo, " stand-alone PV syatem with MPPT function based on fuzzy logic control for remote building application”, International Journal of Power Electronics and Drive Systems (IJPEDS), Vol 10, No 2, June 2019.

[11] Salwa Assahout, Hayat Elaissaoui, Abdelghani El Ougli, Belkassem Tidhaf, Hafida Zrouri, "A Neural Network and Fuzzy Logic based MPPT Algorithm for Photovoltaic Pumping System", International Journal of Power Electronics and Drive Systems (IJPEDS), Vol 9, No 4, December 2018.

[12] Nabil Farah, M. H. N. Talib, Z. Ibrahim, J. M. Lazi, Maaspaliza Azri, "Self-tuning Fuzzy Logic Controller Based on Takagi-Sugeno Applied to Induction Motor Drives", International Journal of Power Electronics and Drive Systems (IJPEDS), Vol 9, No 4, December 2018. 